\title{
Frührehabilitation - ein Stiefkind der Psychiatrie
}

\author{
Early Rehabilitation - A Step Child of Psychiatry
}

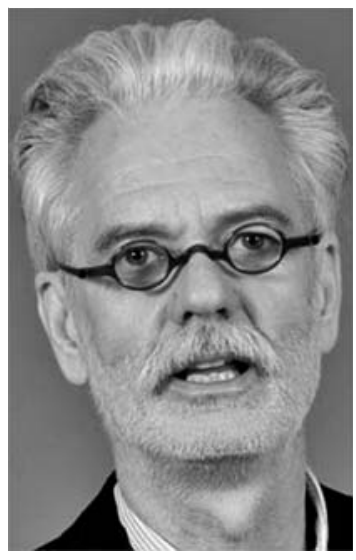

Holger Hoffmann

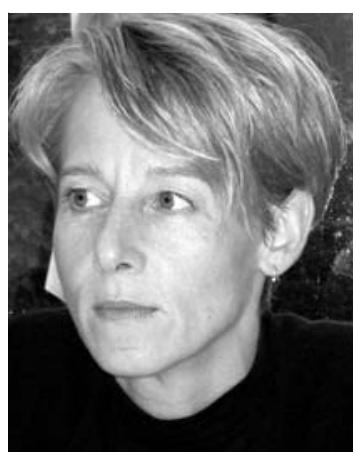

Dorothea Jäckel
Die Inklusion psychisch kranker Menschen in das Arbeitsleben wird nicht nur erschwert durch hohe Zugangsschwellen zur Arbeitsrehabilitation und geringe Wirksamkeit traditioneller arbeitsrehabilitativer Ansätze, sondern auch durch das Fehlen einer psychiatrischen Frührehabilitation. Meist folgt nach Verlust des Arbeitsplatzes eine Phase der Stagnation und des Abwartens, die den Chronifizierungsprozess befördert und eine spätere Reintegration erheblich erschwert oder sogar verhindert $[1,2]$.

Die Mehrzahl der Menschen mit psychischen Störungen befindet sich in psychiatrisch-psychotherapeutischer Behandlung im Rahmen verschiedener Settings (stationär, tagesklinisch, ambulant), in denen eine Identifikation und Bearbeitung ihrer arbeitsbezogenen Problemlagen kaum stattfindet. Zudem wird die Versorgung, statt am aktuellen individuellen Teilhabebedarf orientiert, in fragmentierter und wenig koordinierter Weise angeboten und finanziert. Auch ist in klinischen Leitlinien das nahezu vollständige Fehlen rehabilitativer Aspekte kritisch zu vermerken [3, 4]. Daraus entsteht die paradoxe Situation, dass die Akutbehandlung - obwohl leitlinienkonform durchgeführt - Exklusionsprozesse und frührehabilitative Bedarfe weder in den Blick nimmt noch in die klinische Behandlung integriert.

Die Folge ist, dass 70\% der (teil-)stationär psychiatrisch behandelten PatientInnen im erwerbsfähigen Alter aus dem Erwerbsleben ausgegliedert sind. Dies generiert hohe direkte und indirekte Kosten. Ungeachtet dessen werden berufliche Problemlagen bei psychisch Kranken durch die medizinischen Versorger erst spät im Krankheitsverlauf erkannt und angegangen.

In der Schweiz hat die Invalidenversicherung mit der Einführung von Integrationsmaßnahmen zwar die Möglichkeit geschaffen, früher als bisher arbeitsrehabilitativ zu intervenieren. Damit diese auch wirklich zum Einsatz kommen und Chancen auf Erfolg haben, bedarf es jedoch zusätzlicher, zielgerichteter therapeutischer Interventionen. Integrierte psychotherapeutisch-rehabilitative Konzepte der Frührehabilitation, wie sie beispielsweise in der Neurologie vorliegen, fehlen in der psychiatrischen Arbeitsrehabilitation leider immer noch. Auch die vor einigen Jahren entwickelte medizinisch beruflich orientierte Rehabilitation (MBOR) wird in der Rehabilitationspraxis noch nicht gemäß der Vorgaben durchgeführt [5].

Chefarzt, Direktion

Psychiatrische Rehabilitation und Soteria Bern, Universitäre Psychiatrische Dienste Bern Murtenstrasse 46, Postfach 52 CH-3000 Bern 10, Schweiz hoffmann@spk.unibe.ch

\section{Return to Work}

$\nabla$

Die Return to Work-Raten differieren bei den verschiedenen psychiatrischen Diagnosegruppen. Ihnen gemeinsam ist, dass rehabilitative Interventionen bereits im ersten Monat der Arbeitsunfähigkeit besonders wirksam sind [6-8]. Eine gewisse Zügigkeit ist somit im Rehabilitationsprozess geboten, da bereits nach 6-monatiger Arbeitsunfähigkeit die Wahrscheinlichkeit wieder eine Arbeit aufzunehmen um die Hälfte reduziert ist.

Einen wichtigen Indikator für den Return to Work stellt die Einschätzung der Betroffenen selbst dar $[9,10]$. Frühzeitige Beratung über rehabilitative Möglichkeiten im Zusammenhang mit Versicherungsleistungen und ein nahtloser Einstieg in den Rehabilitationsprozess kann die Eingliederung signifikant verbessern [11]. Für junge Erwachsene beinhaltet Frührehabilitation eine konzeptionelle Ausrichtung auf deren besonderen Bedarfe und spezifischen Lebenssituationen, z.B. durch die Implementierung von Supported Education-Programmen. Außerdem zielt Frührehabilitation darauf ab, Chronifizierungsprozessen durch verfrühte Platzierung in einer WfbM entgegenzusteuern, Invalidisierungsprävention zu betreiben, soziale Exklusionsrisiken zu minimieren sowie die ersten Schritte der Rehabilitationsplanung bereits während der klinischen Behandlung aufzugleisen.

\section{Barrieren und Förderfaktoren der Frührehabilitation \\ $\nabla$}

Versorgungsstrukturen und -angebote können grundsätzlich entweder als Barrieren oder als Förderfaktoren auf die Inklusion wirken. Die Fragmentierung des Rehabilitationssystems, einhergehend mit langen Krankentaggeldzahlungen ohne gleichzeitigem Beginn von ersten Rehabilitationsschritten, stellen eine Barriere für den Return to Work dar. Ebenfalls hemmend wirkt eine klinische Versorgung mit fehlender Ausrichtung auf arbeitsbezogene und psychosoziale Bedarfe. Arbeitsrehabilitation setzt häufig erst spät im Behandlungsprozess, nach Arbeitsplatzverlust und im fortschreitenden Chronifizierungsprozess, ein. Da die Wiedereingliederung aus der Berentung oder ein Wechsel von einer Tätigkeit in der WfbM auf den allgemeinen Arbeitsmarkt nur in Einzelfällen gelingt, sind Entscheidungen hinsichtlich der Wahl geeigneter Interventionen zu einem möglichst frühen Zeitpunkt im Recoveryprozess zu treffen. 
Förderlich und prognostisch günstig wirkt der frühzeitige Zugang in evidenzbasierte Arbeitsrehabilitation nach dem Konzept des Supported Employment [12]. Dessen Wirksamkeit liegt neben der raschen Platzierung auf den allgemeinen Arbeitsmarkt und einer zeitlich unbefristeten Begleitung am Arbeitsplatz in einem Anreizsystem und Dienstleistungspaket für die Arbeitgeber.

Die Implementierung von Screenings in die Routineversorgung zur Identifizierung beruflicher Problemlagen bereits während der klinischen Behandlung im stationären oder tagesklinischen Setting stellt einen wichtigen Förderfaktor dar. Damit Fähigkeitsund Teilhabestörungen überhaupt in den Fokus gelangen und Rehabilitationsbedarfe frühzeitig festgestellt und angegangen werden können, sollte die Dimension der funktionalen Gesundheit (ICF) einbezogen werden. Der frühzeitige Einsatz von rehabilitativen Elementen in die klinische Behandlung kann ein spezifisches vorgängiges Training erforderlich machen, das in dem Konzept der Readiness for Rehabilitation ausgearbeitet ist.

\section{Readiness for Rehabilitation \\ $\nabla$}

Das am Boston University Center for Psychiatric Rehabilitation entwickelte Konzept der Readiness for Rehabilitation [13] knüpft an den Interessen und der Bereitschaft der RehabilitandInnen an, sich in den Rehabilitationsprozess zu involvieren und zu engagieren, um ihr Rehabilitationsziel zu erreichen. Es geht um einen ersten Schritt in der Rehabilitation, in dem gemeinsam eine Basis für die folgenden Schritte in einem konstruktiven Veränderungsprozess gesucht wird. Im Anschluss an das Assessment wird gemeinsam das Rehabilitationsziel entwickelt und festgelegt.

\section{Berner ready@work-Programm \\ $\nabla$}

Das kürzlich gestartete therapeutisch-rehabilitative Berner ready@work-Programm sucht Schnittstellen- und Integrationsprobleme der Versorgung an 3 Stellen zu überwinden. Die Interventionen des 12-32 Wochen dauernden ready@work-Programms richten sich 1 . an psychisch Kranke, die sich noch in stationärer oder teilstationärer psychiatrisch-psychotherapeutischer Behandlung befinden und bei denen mittels eines Screenings berufliche Problemlagen und/oder drohende Exklusion identifiziert wurden, 2. an psychisch Kranke, deren Readiness for Rehabilitation noch wenig entwickelt ist und die, sobald sie Ambivalenzen überwunden, ein Commitment aufgebaut haben und in den Rehabilitationsprozess involviert sind, 3. nahtlos ins Supported Employment und an einen geeigneten Arbeitsplatz in der freien Wirtschaft mit grundsätzlich zeitlich unbefristeter Begleitung wechseln.

Die Finanzierung von ready@work erfolgt durch die Invalidenversicherung und die Krankenkassen. Das Angebot basiert auf dem Konzept der „Funktionalen Gesundheit“ gemäß ICF. Die Überwindung und Kompensation von Funktionseinbußen stehen im Zentrum der Frührehabilitation.

\section{Fazit}

$\nabla$

Frührehabilitation für Menschen mit psychischen Störungen greift einen lange vernachlässigten Bedarf in der Versorgung auf und stellt einen wichtigen Bestandteil einer auf Inklusion ausgerichteten Psychiatrie dar. Sie sucht Barrieren der (Wieder-)Eingliederung zu überwinden und Exklusion zu vermeiden. Frührehabilitation rückt Arbeitswelt und klinische Versorgung zusammen und überwindet die Fragmentierung der beiden konträren Paradigmen der klinischen und rehabilitativen Versorgung.

Ein erster und entscheidender Schritt liegt nun darin, anhand eines definierten Behandlungspfads, PatientInnen mit beruflichen Problemlagen bereits während der klinischen Behandlung über ein Screening zu identifizieren und dann nahtlos in das frührehabilitative Programm aufzunehmen. Klinische Instabilitäten können durch die psychiatrisch-psychotherapeutischen Behandlungselemente aufgefangen und die Präsenzzeiten am Arbeitsplatz schrittweise gesteigert werden.

Das Berner ready@work-Programm verringert einerseits die Schnittstellen zur klinischen Behandlung und andererseits zum allgemeinen Arbeitsmarkt durch das in der Regelversorgung fest implementierte Job Coach Placement, das mit hoher Güte die Supported Employment-Kriterien umsetzt [14].

\section{Literatur}

1 Baer $N$, Frick U, Fasel T. Dossieranalyse der Invalidisierungen aus psychischen Gründen. Typologisierung der Personen, ihrer Erkrankungen, Belastungen und Beratungsverläufe. Forschungsbericht Nr. 6/09. Bern: Bundesamt für Sozialversicherung; 2009

2 Fluder $R$, Salzgeber $R$, Fritschi $T$ et al. Verläufe und Profile von IV-Neurentner/innen 2010. Analysen anhand der SHIVALV-Daten 2005-2010. Forschungsbericht Nr. 10/13. Bern: Bundesamt für Sozialversicherungen; 2014

3 Jäckel WH, Beindorf G, Glattacker M. Berücksichtigung der Rehabilitation in den S3-Leitlinien im Register der Arbeitsgemeinschaft der Wissenschaftlichen Medizinischen Fachgesellschaften e. V. (AWMF). Rehabilitation 2013; 52: $303-306$

4 Schulz H, Büscher $C$, Koch $U$ et al. Leitlinien für die rehabilitative Behandlung von Patienten mit psychischen und psychosomatischen Störungen. Z Psychiatr Psych Ps 2006; 54: 53-64

5 Streibelt $M$, Brünger $M$. Wie viele arbeitsbezogene Leistungen bekommen Patienten mit besonderen beruflichen Problemlagen? Analyse einer repräsentativen indikationsübergreifenden Stichprobe von Rehabilitanden. Rehabilitation 2014; 53: 369 - 375

6 Corbière M, Negrini A, Dewa CS. Mental health problems and mental disorders: Linked determinants to work participation and work functioning. In: Loisel P, Anema JR, eds. Handbook of work disability prevention and management. New York: Springer; 2013: 267-288

7 DRV, eds. Bedeutung psychischer Erkrankungen in der Rehabilitation und bei Erwerbsminderung. Positionspapier der Deutschen Rentenversicherung zur DGPPN-S3-Leitlinie Psychosoziale Therapien bei schweren psychischen Erkrankungen. Berlin: Deutsche Rentenversicherung Bund; 2014

8 Roelen CAM, Norder G, Koopmans PC et al. Employees sick-listed with mental disorders: Who returns to work and when? J Occup Rehabil 2012; 22: $409-417$

9 Blank L, Peters J, Pickvance $S$ et al. A systematic review of the factors which predict return to work for people suffering episodes of poor mental health. J Occup Rehabil 2008; 18: 27 - 34

10 Cornelius LR, van der Klink JJL, Groothoff JW et al. Prognostic factors of long-term disability due to mental disorders: A systematic review. J Occup Rehabil 2011; 21: 259-274

11 Rosen MI, Ablondi K, Black AC et al. Work outcomes after benefits counseling among veterans applying for service connection for a psychiatric condition. Psychiatr Serv 2014; 65: 1426-1432

12 Bond GR, Drake RE. Making the case for IPS supported employment. Adm Policy Ment Health 2014; 41: 69-73

13 Farkas MD, Sullivan-Soydan AP, Gagne C. Introduction to rehabilitation readiness. Boston: Center for Psychiatric Rehabilitation, Boston University; 2000

14 Hoffmann $H$. Berufliche Rehabilitation: First Place, then Train - Pro. Psychiat Prax 2014; 41: 293 - 294 